\title{
State of Health Aware Charge Management in Hybrid Electrical Energy Storage Systems
}

\author{
Qing Xie, Xue Lin, Yanzhi Wang, and Massoud Pedram \\ Department of Electrical Engineering \\ University of Southern California \\ Los Angeles, CA, 90089 \\ \{xqing, xuelin, yanzhiwa, pedram\}@usc.edu
}

\author{
Donghwa Shin, and Naehyuck Chang \\ Department of EECS/CSE \\ Seoul National University \\ Seoul, Korea 151-744 \\ \{dhshin, naehyuck\}@elpl.snu.ac.kr
}

\begin{abstract}
This paper is the first to present an efficient charge management algorithm focusing on extending the cycle life of battery elements in hybrid electrical energy storage (HEES) systems while simultaneously improving the overall cycle efficiency. In particular, it proposes to apply a crossover filter to the power source and load profiles. The goal of this filtering technique is to allow the battery banks to stably (i.e., with low variation) receive energy from the power source and/or provide energy to the load device, while leaving the spiky (i.e., with high variation) power supply or demand to be dealt with by the supercapacitor banks. To maximize the HEES system cycle efficiency, a mathematical problem is formulated and solved to determine the optimal charging/discharging current profiles and charge transfer interconnect voltage, taking into account the power loss of the EES elements and power converters. To minimize the state of health $(\mathrm{SoH})$ degradation of the battery array in the HEES system, we make use of two facts: the SoH of battery is better maintained if (i) the SoC swing is smaller, and (ii) the same SoC swing occurs at lower average SoC. Now then using the supercapacitor bank to deal with the high-frequency component of the power supply or demand, we can reduce the SoC swing for the battery array and lower the SoC of the array. A secondary helpful effect is that, for fixed and given amount of energy delivered to the load device, an improvement in the overall charge cycle efficiency of the HEES system translates into a further reduction in both the average $\mathrm{SoC}$ and the $\mathrm{SoC}$ swing of the battery array. The proposed charge management algorithm for a Li-ion battery - supercapacitor bank HEES system is simulated and compared to a homogeneous EES system comprised of Li-ion batteries only. Experimental results show significant performance enhancements for the HEES system, an increase of up to $21.9 \%$ and $4.82 x$ in terms of the cycle efficiency and cycle life, respectively.
\end{abstract}

Keywords: hybrid electrical energy storage system, charge management, state of health.

\section{INTRODUCTION}

The generation and consumption of electrical energy typically do not match with each other. Electrical energy storage (EES) systems can increase the availability of the electrical energy, mitigate the supply-demand mismatches, and reduce the generation capacity required to meet the peak-power demand. Performance metrics of EES systems include cycle efficiency, cycle life, energy density, power capacity, cost per unit capacity, environmental effect including end-of-life disposal cost. As none of existing EES element can satisfy all the required performance metrics such as high power/energy density, low cost/weight per unit capacity, high round-trip efficiency, and long cycle life. This limitation discourages quick and wide spread of large-scale EES systems.

This research is sponsored in part by a grant from the National Science Foundation, and by the Brain Korea 21 Project, IC Design Education Center (IDEC), and Mid-career Researcher Program through NRF grant funded by the MEST (No. 2010-0017680).
A hybrid EES (HEES) system, on the other hand, comprises of heterogeneous EES elements, where each type has its unique strengths and weaknesses. The appropriate control policies can take advantage of the strengths of individual type of elements while hiding their shortcomings. In this way, we can achieve superior overall performance metrics that overcome the limitation of individual EES components [1] [4]. Among these performance metrics, the cycle life of the EES elements is one of the most important metrics that should be considered by the designers of the EES system. The cycle life is directly related to the state of health $(\mathrm{SoH})$, which is defined as the ratio of full charge capacity (FCC) of a cycle-aged EES element to its designed capacity (DC). This metric captures the general condition of the EES elements and their ability to store and deliver energy compared to its initial state (i.e., compared to a fresh new EES element). Some researchers have worked on extending the lifetime of EES elements [5] [8]. However, they only focus on either a single EES element or a homogeneous EES system, which consists of a single type EES element array.

Unlike a single element or a homogeneous EES system, the cycle life of the EES elements in a HEES system is largely dependent on the HEES charge management policy. This is the first paper that introduces the cycle life of EES elements in a HEES system and develops a charge management policy, including allocation, replacement and migration, to prolong the cycle life of EES elements. We deal with extending the cycle life of the HEES system as well as improving the cycle efficiency. In this paper, we first introduce a generalized architecture of the HEES system and build the corresponding electrical circuit models for power converters, battery elements and supercapacitor elements. We adopt the cycle life model of [9] and determine the $\mathrm{SoH}$ degradation rate with respect to different average state of charges (SoCs, defined as available capacity remaining in the battery, expressed as a percentage of the rated capacity) and $\mathrm{SoC}$ swings (defined as $\mathrm{SoC}$ change during a charging / discharging cycle). We take into account the power dissipation on internal resistance of EES elements, power loss due to power converters and rate capacity effect of the batteries elements.

We simplified the target HEES system to a two-bank architecture in order to focus on the idea of SoH-aware charge management, i.e., using supercapacitor banks as buffer to shave the spiky portion of the source or load profiles so that battery banks can stably receive energy from the power sources or provide energy to the load devices. During the charging/discharging process, we achieve high cycle efficiency by determining the optimal CTI voltage and subsequently the charging or discharging currents for battery and supercapacitor banks through a ternary search. Besides improving the cycle efficiency, we also reduce the SoC swing and average SoC of battery arrays, which alleviates the $\mathrm{SoH}$ degradation. We implement our algorithm on a sample HEES system and compare with a typical homogeneous EES system. Experimental results demonstrate significant improvement up to $21.9 \%$ and $4.82 \mathrm{x}$ in terms of cycle efficiency and cycle life, respectively. 


\section{BACKGROUND}

\section{A. Related Work}

The $\mathrm{SoH}$ of the batteries is hard to estimate because it is related to a capacity fading effect (i.e., SoH degradation) which is a result of long-term electrochemical reaction. The capacity fading is related with carrier concentration loss and internal impedance growth in the batteries. These effects strongly depends on the operating condition of the battery such as charging and discharging current, number of cycles, SoC swing, average SoC and operation temperature [10] [12]. The characterization of the battery cell requires timeconsuming experiments. Thus, mathematical models help us to reduce the time complexity in estimating the $\mathrm{SoH}$ degradation. Electrochemistry-based models [13] [15] are generally accurate but not easy to implement. The SoH degradation model in [9], showing a good match with real data in [12], is suitable for HEES systems.

Appropriate control of charging and discharging process is a representative way to enhance the cycle life of the batteries. Typical Li-ion batteries are usually charged through two continuous steps, constant-current step (CC) and constant-voltage step (CV). Each step has an effect on the charging time and battery lifetime. Several techniques have been introduced to improve the cycle life [5] [8]. However, although these works are effective, they are all focused on the single type of EES elements or homogeneous EES systems.

\section{B. HEES System Architecture and Charge Management}

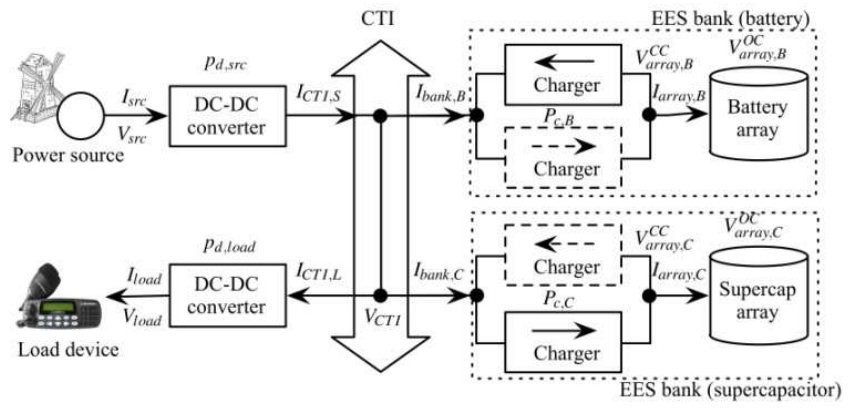

Figure 1: Conceptual diagram of charge management problem.

Figure 1 shows the simplified diagram of the HEES system architecture [1]. The HEES system is comprised of multiple heterogeneous EES banks connecting to each other through Charge Transfer Interconnect (CTI). An EES bank contains an EES element array, a discharging control charger and a charging control charger. Each EES array is composed of multiple homogeneous EES elements with the same SoC since a single EES element has low voltage rating and small energy capacity. Of these two chargers, only one of them can be turned on at a time to avoid simultaneous charging and discharging of an EES array.

For simplicity, in Figure 1, we only show two representative EES banks: a battery bank and a supercapacitor bank. At time $t$, we use $V_{\text {array,B }}^{O C}(t)$ and $V_{\text {array, } B}^{C C}(t)$ to denote open circuit terminal voltage $(\mathrm{OCV})$ and closed circuit terminal voltage $(\mathrm{CCV})$ of battery array, respectively. These two voltages are generally not equal to each other due to the internal resistances of the battery array. The relation between $V_{\text {array,B }}^{O C}(t)$ and $V_{\text {array,B }}^{C C}(t)$ is given in Section III.A. We denote the array current between the battery array and its chargers by $I_{\text {array, } B}(t)$ and bank current between CTI and corresponding charger by $I_{\text {bank }, B}(t)$. The $I_{\text {array }, B}(t)$ and $I_{\text {bank }, B}(t)$ can be positive (current goes from CTI to battery array) or negative (current goes from battery array to CTI). The power loss of corresponding control charger is denoted by $P_{c, B}(t)$ and is a function of its input and output voltages and currents, as shown in Section III.B. Similar notations are also used for the supercapacitor bank by replacing subscript $B$ to $S$. The load devices and the power sources are connected to HEES system through DC-DC converters to maintain the voltage-level compatibility. We denote the power loss of DC-DC converters $P_{d, l o a d}(t)$ and $P_{d, s r c}(t)$, the voltages and currents of load device and power source by $V_{\text {load }}(t), I_{\text {load }}(t)$ and $V_{s r c}(t), I_{s r c}(t)$, and the current through CTI by $I_{C T I}(t)$. The chargers have internal feedback control loops to maintain their stable outputs, and a centralized HEES controller (not in the figure) to give the set points. In addition, the HEES controller has an outer feedback loop monitoring the CTI voltage, denoted by $V_{C T I}(t)$. This setup allows us to maintain both the charging currents and the CTI voltage.

\section{SYSTEM MODEL}

\section{A. EES Element Array}

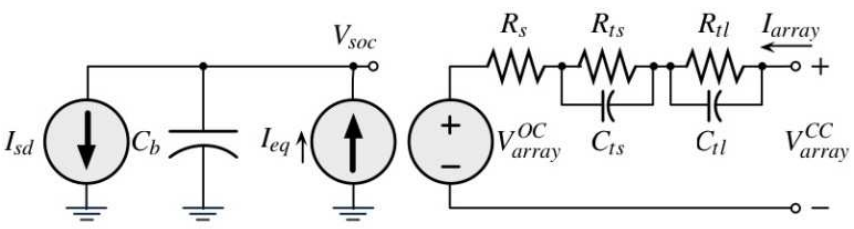

Figure 2: Li-ion battery circuit model.

This paper addresses charge management problem in HEES system with two representative EES elements: Li-ion battery and supercapacitor. We use a circuit model in [16] for the Li-ion battery, which is suitable for developing a mathematical formulation. The Figure 2 shows the circuit model for the Li-ion battery. The $V_{S o C}$ is the voltaic expression of the SoC of the battery, which is related to the OCV of battery through a non-linear relation, given by:

$V_{\text {array }}^{O C}(t)=b_{1} e^{b_{2} V_{S o C}(t)}+b_{3} V_{S o C}^{3}(t)+b_{4} V_{S o C}^{2}(t)+b_{5} V_{S o C}(t)+b_{6}$, (1)

where these $b_{i}$ are empirical parameters [17]. The relation between the battery array OCV and CCV (for charging process), denoted by $V^{O C}$ and $V^{C C}$, is given by:

$$
V_{\text {array }}^{C C}(t)=V_{\text {array }}^{O C}(t)+V_{t l}(t)+V_{t s}(t)+I_{\text {array }}(t) \cdot R_{s}
$$

where $V_{t l}(t)$ and $V_{t s}(t)$ are the voltage drops across the internal capacitances, $I_{\text {array }}(t)$ is the array current and $R_{S}$ is the internal series resistance. Similar relations can be applied to supercapacitor arrays in which the internal capacitances are negligible.

The rate capacity effect of batteries describes the fact that the available discharging time of a battery element array is strongly dependent on the array discharging current. The Peukert's Law [18] shows that the empirical relation between the available discharging time and discharging current can be expressed as $C_{p}=I^{\gamma_{B, d}} t$, where $C_{p}$ is the nominal capacity (in unit of $A \cdot h$, usually defined as the capacity at one-ampere discharge rate), $I$ is the actual array discharging current, $t$ is the discharging time and $\gamma_{B, d}$ is the Peukert constant (typically between 1.1 and 1.3 for discharging). Therefore, for a discharging process, the power drawn from battery banks to provide $V_{\text {array }, B}^{O C}(t) \cdot I_{\text {array }, B}(t)$ of power to the circuits is given by:

$$
P_{\text {drawn }, B}(t)=V_{\text {array }, B}^{O C}(t) \cdot I_{e q, B}(t)=V_{\text {array }, B}^{O C}(t) \cdot I_{\text {array }, B}^{\gamma_{B, d}}(t),
$$

where $I_{e q, B}(t)$ is the equivalent current inside battery. The rate capacity effect causes power loss during charging process in a similar way. The power can be store into battery bank, while being charged with $V_{\text {array }, B}^{O C}(t) \cdot I_{\text {array, } B}(t)$ of power, is given by:

$$
P_{\text {gain }, B}(t)=V_{\text {array }, B}^{O C}(t) \cdot I_{e q, B}(t)=V_{\text {array }, B}^{O C}(t) \cdot I_{\text {array }, B}^{\gamma_{B, c}}(t),
$$

where $\gamma_{B, C}$ is the Peukert constant for charging, typically between 0.8 0.9. Similar relations can also be applied to supercapacitor arrays 
except that the rate capacity effect is negligible, i.e., $\gamma_{C, C}=\gamma_{C, d}=1$ in (3) and (4).

\section{B. Power Converters}

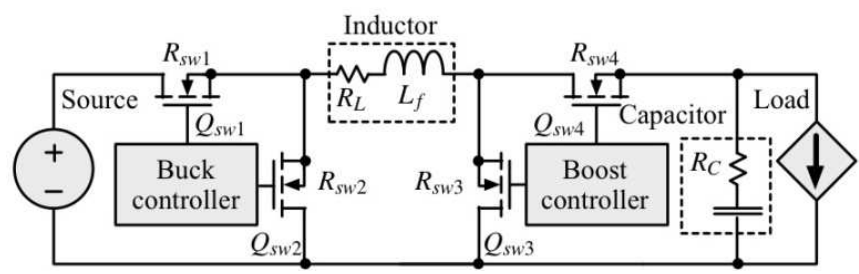

Figure 3: Buck-boost converter circuit model [19].

A charger is a switching converter which can regulate its output current to a desired value. The target HEES uses PWM (pulse width modulation) 4-switches buck-boost switching converters for a charger, as shown in Figure 3. The input voltage, input current, output voltage and output current of the charger are denoted by $V_{i n}$, $I_{\text {in }}, V_{\text {out }}$ and $I_{\text {out }}$, respectively. Depending on the relation between $V_{\text {in }}$ and $V_{\text {out }}$, the charger has two operating modes: the buck mode (if $V_{\text {in }}>V_{\text {out }}$ ) and otherwise the boost mode. The power loss $P_{c}$ consists of three components: conduction loss $P_{c d c t}$, switching loss $P_{s w}$ and controller loss $P_{c t r l}$ [19]. The power loss $P_{c}$ is given by:

$$
P_{c}=P_{c d c t}+P_{s w}+P_{c t r l},
$$

The buck mode power loss components are given by:

$$
\begin{aligned}
& P_{c d c t}=I_{\text {out }}{ }^{2} \cdot\left(R_{L}+D \cdot R_{s w 1}+(1-D) \cdot R_{s w 2}+R_{s w 4}\right) \\
& +1 / 12 \cdot(\Delta I)^{2} \cdot\left(R_{L}+D \cdot R_{s w 1}+(1-D) \cdot R_{s w 2}+R_{s w 4}+R_{C}\right), \\
& P_{s w}=V_{\text {in }} \cdot f_{s} \cdot\left(Q_{s w 1}+Q_{s w 2}\right), \\
& P_{c t r l}=V_{\text {in }} \cdot I_{\text {controller }},
\end{aligned}
$$

where $D=V_{\text {out }} / V_{\text {in }}$ is the PWM duty ratio and $\Delta I=V_{\text {out }}$. $(1-D) /\left(L_{f} \cdot f_{s}\right)$ is the maximum current ripple; $f_{s}$ is the switching frequency; $I_{\text {controller }}$ is the operating current of the controller; $R_{L}$ and $R_{C}$ are the equivalent series resistances of the inductor $L$ and capacitor $C$, respectively; $R_{S w i}$ and $Q_{s w i}$ are the turn-on resistance and gate charge of $i^{\text {th }}$ MOSFET switch in Figure 3, respectively. The boost mode power loss components are given by:

$$
\begin{aligned}
& P_{c d c t}=\left(\frac{I_{\text {out }}}{1-D}\right)^{2}\left(R_{L}+D R_{s w 3}+(1-D) R_{s w 4}+R_{s w 1}+D(1-D) R_{C}\right) \\
& +1 / 12(\Delta I)^{2} \cdot\left(R_{L}+D \cdot R_{s w 3}+(1-D) \cdot R_{s w 4}+R_{s w 1}+(1-D) \cdot R_{C}\right), \\
& P_{s w}=V_{\text {out }} \cdot f_{s} \cdot\left(Q_{s w 3}+Q_{s w 4}\right), \\
& P_{c t r l}=V_{\text {in }} \cdot I_{\text {controller }},
\end{aligned}
$$

where $D=1-V_{\text {in }} / V_{\text {out }}$ and $\Delta I=V_{\text {in }} \cdot D /\left(L_{f} \cdot f_{s}\right)$ in this case.

We use the same model for the DC-DC converters for load devices and power source. The power loss of the DC-DC converter, denoted by $P_{d}$, is also given by (5) (7) with different set of parameters and input/output voltages and currents.

\section{SoH Degradation Model}

The rate of SoH degradation depends on the average SoC level and SoC swing [9]. We consider a time interval of $[0, \tau]$ and calculate the average and standard deviation of $\mathrm{SoC}$ as:

$$
\begin{aligned}
& S o C_{a v g}=\int_{0}^{\tau} \operatorname{SoC}(t) d t / \tau, \\
& S o C_{d e v}=2 \sqrt{3 \int_{0}^{\tau}\left(\operatorname{SoC}(t)-S_{o} C_{a v g}\right)^{2} d t / \tau},
\end{aligned}
$$

$S o C_{d e v}$ is normalized to have the value of 1.0 for the full $100 \%$ depth of discharge cycle, i.e. SoC ranges from 1.0 down to 0 and back to
1.0. $S o C_{d e v}$ can be used to approximately describe the SoC swing if the battery SoC increases and decreases stably. The battery may have multiple charges and discharges due to the different magnitude of operation current during the time interval $\tau$. Thus we compute the effective throughput number of cycle $N$ as follows:

$$
N=\int_{0}^{\tau}|I(t)| d t / 2 Q_{n}
$$

where $I(t)$ is the charging/discharging current of the battery, which is $I_{e q, B}(t)$ in (3)(4), $Q_{n}$ is the nominal charge capacity of the battery. The SoH degradation during this time interval, accounting the average SoC level and SoC swing, is given by:

$$
\begin{aligned}
& L_{1}=K_{c o} \cdot N \cdot \exp \left[\left(S o C_{d e v}-1\right) \cdot T_{r e f} / K_{e x} / T_{B}\right]+0.2 \tau / \tau_{\text {life }}, \\
& L_{2}=L_{1} \cdot \exp \left[4 K_{S o C} \cdot\left(S o C_{a v g}-0.5\right)\right] \cdot(1-L), \\
& L_{3}=L_{2} \cdot \exp \left[K_{T} \cdot\left(T_{B}-T_{r e f}\right) \cdot T_{r e f} / T_{B}\right]
\end{aligned}
$$

where the empirical constants $K_{c o}, K_{e x}, K_{S o C}$ and $K_{T}$ are battery specific, $T_{r e f}$ and $T_{B}$ is the reference battery temperature and operation battery temperature, respectively, and $\tau_{\text {life }}$ is the calendar life to $80 \% \mathrm{SoH}$. The $\mathrm{SoH}$ degradation after $M$ time intervals is:

$$
L(M)=\sum_{m=1}^{M} L_{3}(m)
$$
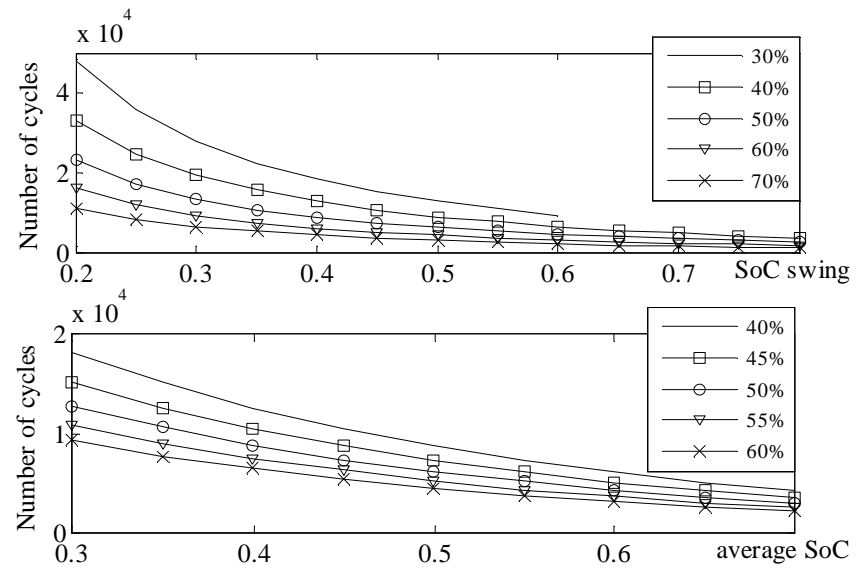

Figure 4: SoH degradation versus $\mathrm{SoC}$ swing (at different average $\mathrm{SoC}$ levels) and average SoC (at different SoC swings).

In (11), the normalized SOH degradation $L$ will change over the life of battery from 0 (brand new) to 1.0 (no capacity left). Typically, the value of $L=0.2$, giving nominally $80 \%$ charge capacity, can be used as a measure of end of useful life. The relations of $\mathrm{SoH}$ degradation versus SoC swing and average SoC level is shown in Figure 4. A cycle is defined as a charging process and a discharging process right after it (i.e., SoC ramps up then back to original value). We can vary the duration of a cycle to achieve different average SoC levels and SoC swings. We repeat cycling until the battery reaches $L=0.2$ and record the total number of cycles at that time. The results are shown in Figure 4. There are three important observations from Figure 4: higher SoH degradation rate is caused by 1) bigger SoC swing, 2) larger average SoC level in each cycle, and 3) the cycle life improvement has a super-linear relation with respect to the reduction of SoC swing and average SoC. We propose our near-optimal charge management algorithm based on these three observations.

\section{State of Health-Aware Charge Management}

\section{A. Problem Statement}

State of health-aware charge management problem in HEES systems is to find CTI voltage and charging/discharging current profiles for all EES banks in a given HEES system, aiming to 
improve both the cycle life of the EES arrays (mainly battery arrays) and overall cycle efficiency of the entire system. In HEES systems, battery arrays have higher energy capacity but relatively lower power capacity. Thus we determine the target charging and discharging rate at which we charge or discharge the battery arrays continuously and stably, considering the battery properties, load devices and power source characteristics. We use the supercapacitor arrays as buffers of battery arrays because they have strengths of high power capacity, superior cycle efficiency and long cycle life but weakness of low energy capacity. We maintain the desired charging currents of the battery banks in charging process, allowing the supercapacitor banks to accept the extra power from power source if the incoming power rate is higher than the target charging rate. Similar strategy is also taken during the discharging process, we let the supercapacitor banks compensate power shortage caused by high load demand and get charged from battery banks to maintain their SoC when the load demand is low.

The charge management problem in HEES system is constrained by energy conservations. As shown in Figure 1, for the charging process, the input power follows:

$$
P_{s r c}(t)=V_{s r c}(t) \cdot I_{s r c}(t)=P_{d, s r c}(t)+V_{C T I}(t) \cdot I_{C T I}(t),
$$

where the current through CTI, $I_{C T I}(t)$, consists of two parts:

$$
I_{C T I}(t)=I_{\text {bank }, C}(t)+I_{\text {bank }, S}(t) .
$$

In (13), $I_{\text {bank }, S}(t)$ can be positive (from CTI to supercapacitor banks) or negative (from supercapacitor banks to CTI), depending on whether supercapacitor banks is used to store excessive energy from source or provide energy to battery banks. For battery banks and supercapacitor banks, we also have:

$$
\begin{aligned}
& V_{C T I} \cdot I_{\text {bank }, S}(t)=P_{c, S}(t)+V_{\text {array }, S}^{C C}(t) \cdot I_{\text {array }, S}(t), \\
& V_{C T I} \cdot I_{\text {bank }, B}(t)=P_{c, B}(t)+V_{\text {array }, B}^{C C}(t) \cdot I_{\text {array }, B}(t) .
\end{aligned}
$$

The relations of OCV and CCV and rate capacity effect are given in (2) (4). Similar relations also hold for discharging process. The overall cycle efficiency of HEES system has two components (charging efficiency and discharging efficiency), given by:

$$
\begin{aligned}
& \eta_{c}=\int_{\tau_{c}}\left[P_{\text {gain }, S}(t)+P_{\text {gain }, B}(t)\right] d t / \int_{\tau_{c}} P_{s r c}(t) d t, \\
& \eta_{d}=\int_{\tau_{d}} P_{\text {load }}(t) d t / \int_{\tau_{d}}\left[P_{\text {drawn }, S}(t)+P_{\text {drawn }, B}(t)\right] d t,
\end{aligned}
$$

and the cycle efficiency is the product of these two, i.e., $\eta=\eta_{c} \eta_{d}$.

The SoH-aware charge management problem can be formally described as following: for a given HEES system, power sources and load devices, we want to find operation current profiles $I_{\text {array,S }}(t)$ for supercapacitor banks and $I_{\text {array,B }}(t)$ for battery banks, such that we can maximize cycle efficiency given by (15), and minimize the SoH degradation for battery array in (11). The supercapacitor array typically has very long cycle life so its $\mathrm{SoH}$ degradation is not considered in this paper.

\section{B. Optimal Operation Condition Search}

At time $t$, the source voltage and current, $V_{s r c}(t)$ and $I_{s r c}(t)$, and SoC of the EES array, which is used to determine $V_{\text {array }}^{O C}(t)$ using (1), are given for a charging process. We omit the time $t$ in following text for convenience. In Figure 5, given $V_{C T I}$, we can calculate CTI current $I_{C T I}$ from (12), and further determine array charging current $I_{\text {array }}$ and equivalent current inside EES array $I_{e q}$ from (2)(4)(14). The instantaneous charging efficiency is a function of $V_{C T I}$, given by:

$$
\eta_{i c}\left(V_{C T I}\right)=P_{\text {gain }}\left(V_{C T I}\right) / P_{s r c} .
$$

Assuming $\eta_{i c}\left(V_{C T I}\right)$ in (16) is a quasi-concave function of $V_{C T I}$, we

\begin{tabular}{|c|c|c|c|c|c|}
\hline \multirow{2}{*}{ Source $\quad I_{s r c}$} & \multirow{2}{*}{$\begin{array}{c}\text { DC-DC } \\
\text { Converter }\end{array}$} & $I_{C T I}$ & \multirow[b]{2}{*}{ Charger } & $I_{\text {array }}$ & \multirow{2}{*}{$\sum_{\mathrm{EES}} I_{e q}$} \\
\hline & & & & & \\
\hline
\end{tabular}
perform a ternary search within the feasible region of CTI voltage to find $V_{C T I}^{o p t}$ and subsequently $I_{C T I}^{o p t}$ and $I_{\text {array }}^{o p t}$, such that $\eta_{i c}\left(V_{C T I}^{o p t}\right)$ achieves its maximum value [3]. The search algorithm converges in a logarithmic time with respect to $V_{C T I}$ precision. The simulation results validate the quasi-concavity assumption. In the similar way, one can easily solve the discharging process.

Figure 5: Schematic of optimal operation condition search.

This proposed online optimal operation condition search (OOCS) algorithm guides us in determining the charging (discharging) current and CTI voltage according to the source (load) characteristics and SoC of the EES arrays. Since the cycle efficiency of battery bank can vary from $50 \%$ to $70 \%$ (depending on the charging or discharging current), operating the battery bank near the optimal operation condition can significantly improve the cycle efficiency, which in turn benefits the cycle life.

The proposed OOCS algorithm is still solvable in polynomial time for the HEES system with multiple banks with some proper charge allocation and replacement algorithms [4]. Without loss of generality, we perform OOCS algorithm to one battery bank and one supercapacitor bank HEES system in this paper.

\section{Crossover Filter}

Battery-only EES systems typically do not perform well if there are high peaks in power supply and demand profile. The peaks cause serious rate capacity effect, which largely hurt the cycle efficiency and cycle life of the battery arrays. Unfortunately, the pulsed load profiles (e.g., radio receiver and transmitter) are very common and the typical renewable power sources, such as windmills, may generate high power fluctuation. The supercapacitor arrays, normally having high power capacity and cycle efficiency, can efficiently deal with the high-frequency component of the power profile.

Thus we apply a crossover filter to the source (load) to separate the high frequency component and the low frequency component. More precisely, we first observe the source and load profile for a time $\tau_{o}$ and then apply the crossover filter to the observed profile. The low frequency component of source profile, for a charging process, denoted by $\hat{V}_{s r c}(t)$ and $\hat{I}_{s r c}(t)$, is given by:

$$
\begin{aligned}
& \hat{V}_{s r c}(t)=\text { Crossover }_{\text {Low }}\left(V_{\text {src }}\left(t^{\prime}\right), t^{\prime} \in\left[t-\tau_{o}, t\right]\right), \\
& \hat{I}_{\text {src }}(t)=\text { Crossover }_{\text {Low }}\left(I_{\text {src }}\left(t^{\prime}\right), t^{\prime} \in\left[t-\tau_{o}, t\right]\right),
\end{aligned}
$$

where the $V_{s r c}\left(t^{\prime}\right)$ and $I_{s r c}\left(t^{\prime}\right)$ are the observed source (load) profile and Crossover $_{\text {Low }}$ is to take the low frequency part of the input signal. Consequently, $\hat{V}_{s r c}(t)$ and $\hat{I}_{s r c}(t)$ provide a smooth source (load) profile, removing the short-term fluctuation and keeping the long-term trend.

\section{Near-optimal Charge Management Algorithm}

In this section, we elaborate our near-optimal charge management algorithm to determine the near-optimal operation condition, which consists of the CTI voltage and EES arrays charging or discharging currents. We solve the charging process as an example. In the similar way, one can easily solve the discharging process.

\section{1) Target Optimal Operation Condition}

We can find a target optimal operation condition for the battery banks in our HEES system, based on the low frequency components of the source power profile obtained using the crossover filter. We assume battery array in Figure 5 and apply the OOCS algorithm: 


$$
\left[\hat{V}_{C T I}^{t a r}(t), \hat{I}_{\text {array }, B}^{t a r}(t)\right]=\operatorname{OOCS}\left(\hat{V}_{s r c}(t), \hat{I}_{s r c}(t), V_{\text {array }, B}^{O C}(t)\right)
$$

The target optimal operation condition is the desired operation condition for battery banks, maximizing the cycle efficiency but ignoring fluctuations of the power supply and demand. The proposed algorithm operates battery bank at near-optimal operation condition for most of the time with the help of the supercapacitor bank.

\section{2) Instantaneous Optimal Operation Condition}

At time $t$, we perform the OOCS algorithm to the instantaneous source power as well and get the optimal operation condition as:

$$
\left[V_{C T I}^{i n s}(t), I_{\text {array }, B}^{\text {ins }}(t)\right]=\operatorname{OOCS}\left(V_{s r c}(t), I_{s r c}(t), V_{\text {array }, B}^{O C}(t)\right)
$$

The instantaneous optimal operation condition is to maximize the instantaneous charging efficiency when the power source charges battery bank only. Note that this optimal condition is affected by the source power fluctuations and does not reflect the overall optimal operation condition for the entire HEES system since the supercapacitor bank is not taken in account in (19).

\section{3) Near-optimal Charge Management Algorithm}

The key idea of the algorithm is to use supercapacitor bank as the buffer of the battery bank. When charging the battery bank, we allocate part of the incoming power to the supercapacitor bank if the incoming power is very high, in order to mitigate the loss caused by rate capacity effect. However, the supercapacitor bank typically has limited energy capacity. It may result in undesirable situation since the supercapacitor bank may be fully charged very quickly and fail to get charged from the power source any more. We overcome this problem by performing charge migration from the supercapacitor bank to the battery bank when the instantaneous charging current is lower than the target charging current and the SoC of the supercapacitor array is high enough. Similarly, we avoid fully discharging the supercapacitor bank by charging it at the beginning of the charging process.

Based on this key idea, we propose the following near-optimal operation algorithm. We first charge the supercapacitor bank until the supercapacitor array has a certain level of SoC, which is HEES system and source (load) specific and can be determined by their statistical information. We determine the two sets of the optimal operation conditions for battery bank at time $t$ : the target and instantaneous operation conditions. We compare $\hat{I}_{\text {array,B }}^{\text {tar }}(t)-$ $I_{\text {array,B }}^{\text {ins }}(t)$ with a positive threshold value $\varepsilon$, and perform appropriate actions such that:

- $I_{\text {array }, B}^{\text {ins }}(t)-\hat{I}_{\text {array }, B}^{\text {tar }}(t)>\varepsilon$, i.e., the instantaneous optimal battery array charging current is higher than the target optimal battery array charging current. It is usually caused by high incoming power. In this case, we charge the battery with the target array charging current $\hat{I}_{\text {array, }}^{\text {tar }}(t)$ and store the excessive power from power source to supercapacitor bank.

- $I_{\text {array }, B}^{\text {ins }}(t)-\hat{I}_{\text {array }, B}^{\text {tar }}(t)<-\varepsilon$, i.e., the instantaneous optimal battery array charging current is lower than the target optimal battery array charging current, which is typically due to the low incoming power. In this case, both of power source and supercapacitor bank are charging the battery bank, maintaining the value of battery array charging current to be $\hat{I}_{\text {array, }}^{\text {tar }}(t)$.

- $\left|I_{\text {array }, B}^{\text {ins }}(t)-\hat{I}_{\text {array, } B}^{\text {tar }}(t)\right|<\varepsilon$, the two optimal battery array charging current match within the threshold value. We can simply charge battery bank with instantaneous optimal array charging current $I_{\text {array }, B}^{\text {ins }}(t)$.

The above policy is summarized in Algorithm 1. The proposed algorithm for HEES system has two distinct advantages. First, we have a supercapacitor bank in the HEES systems that can store the excessive energy from power source or provide extra energy to the load devices. Thus the SoC swing and average SoC level of the battery array can be reduced naturally. Second, a HEES system is superior to a homogeneous EES system when supporting the same load devices and the same minimum SoC requirement for future load demand. The HEES system with the proposed algorithm can achieve higher overall cycle efficiency than that of the homogeneous EES system because of the near-optimal choice of CTI voltage, charging/discharging currents and high cycle efficiency of the supercapacitor bank. In other words, the HEES system needs less input energy to support the same load demand and thereby further reduce the SoC swing and average SoC level of the battery array.

Recall the observations we made from Figure 4, the cycle life of the battery array can be extended if we reduce the SoC swing and the of the battery array. There is super-linear relation between the cycle life improvement with respect to reduction of SoC swing and the average SoC level. Thus the proposed algorithm can further boost up the cycle life of the battery array.

Discharging process is similar to the charging process, but the charge is moving from the EES banks to load devices. We also set a target optimal battery array discharging current and try to maintain it as much as we can during the whole discharging process. We discharge the supercapacitor bank to compensate the power shortage if the load devices require larger current than the target battery discharging current. However, we need to maintain the $\mathrm{SoC}$ of the supercapacitor array since it has small energy capacity and may be fully discharged very quickly. We charge the supercapacitor array using the excessive current when the target battery discharging current is greater than the current required by the load devices to maintain the $\mathrm{SoC}$ of the supercapacitor array.

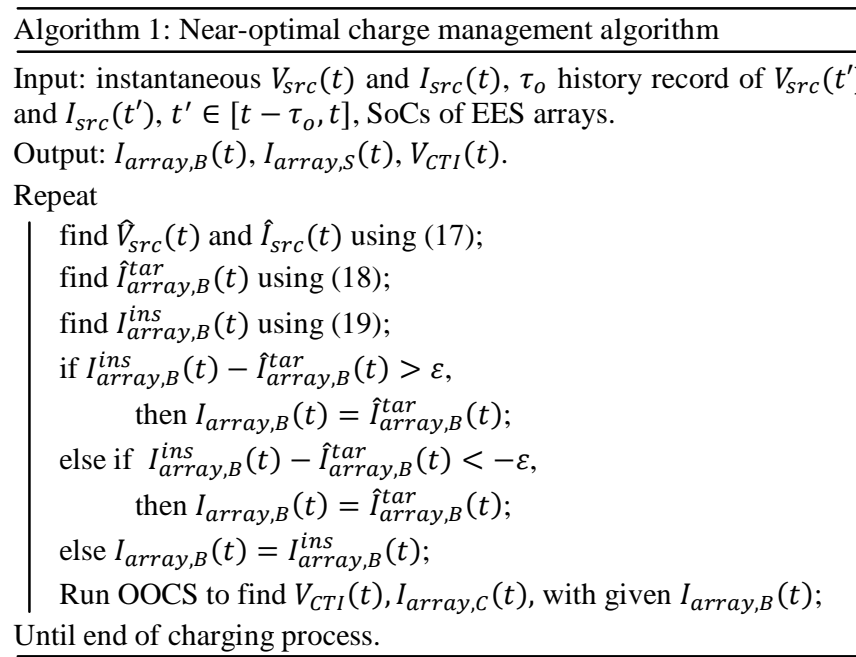

The capacity of the supercapacitor array affects the improvement of the battery array cycle life and the HEES system cycle efficiency. A larger supercapacitor array can enhance both of the two metrics with expense of high capital cost. Thus, we should determine the optimal capacity of supercapacitor array based on the statistical information of the source/load profiles.

\section{EXPERIMENTAL RESULTS}

We implement our near-optimal SoH-aware charge management algorithm on a typical HEES system consisting of a $1 \mathrm{Ah} \mathrm{Li-ion}$ battery, of which the OCV varies from $2.9 \mathrm{~V}$ to $4.2 \mathrm{~V}$, depending on the SoC level, and a $100 \mathrm{~F}$ supercapacitor. We compare the cycle life and overall cycle efficiency of our HEES system with the baseline system, which is a battery-only EES system using the same battery. 
We test our proposed algorithm with two sets of source and load profiles. In the first set, we use a pulse source profile (duty ratio of $30 \%$, average power of $2.7 \mathrm{~W}$ ) and a pulse load profile (duty ratio of $25 \%$, average power of $2 \mathrm{~W}$ ). We use a sinusoidal wave source profile (average power of $4.5 \mathrm{~W}$ ) and a sinusoidal wave load (average power of $4 \mathrm{~W}$ ) profile in the second set. A cycle starts from a charging process and then discharges to provide power to the load. Hence the battery SoC, starting from the initial value, will first ramp up and then down to this value. We test various different durations of the discharging process (i.e., different load energy demands) for each set of experiments so that we can achieve different battery SoC swings and average SoC levels. We let the HEES system have the same minimum SoC requirement for battery and the same duration of discharging process as those of the baseline system. However, the charging process for HEES system may be shorter than that of the baseline system due to higher cycle efficiency of HEES system.
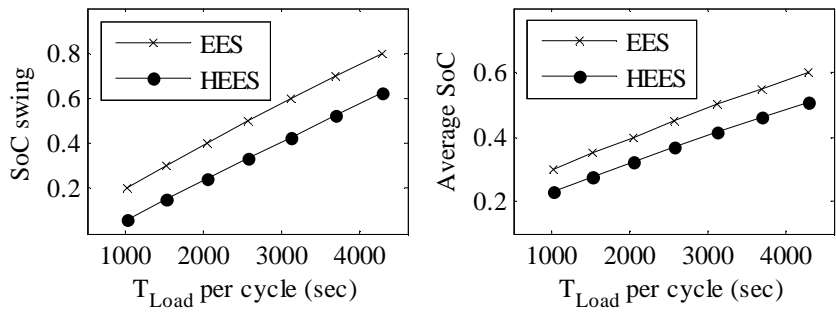

Figure 6: SoC swing and average SoC of battery-only EES system and HEES system vs duration of pulse load profile per cycle.
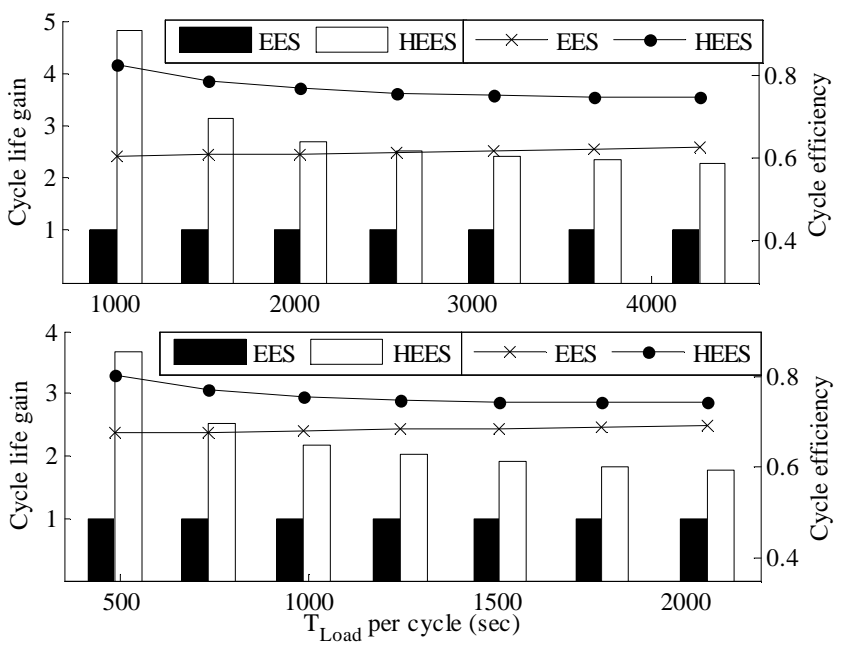

Figure 7: Normalized cycle life gain (bars) and cycle efficiency improvement (curves) with different sources and loads profile (a - pulse, $b$ - sine wave).

Figure 6 shows that our proposed algorithm can effectively reduce the $\mathrm{SoC}$ swing (a) and average $\mathrm{SoC}$ level (b) in each cycle in the HEES system, compared to the baseline system. Two factors contribute to this reduction. First, part of the energy is allocated and then extracted from the supercapacitor bank. Hence the battery bank receives and provides less energy. Second, the battery bank stably gets charged and discharged after the supercapacitor bank shaves the peaks of the power supply and demand. Thus the battery bank itself is more efficient in the HEES system.

We repeat simulation for tens of thousands of cycles until the battery SoH degradation in (11) reaches 0.2 . The results of the cycle life gain (normalized to baseline system) and overall cycle efficiency are shown in Figure 7. We demonstrate significantly normalized cycle life and overall cycle efficiency improvement in Figure 7. For the pulse source and load profile, the cycle life of HEES system is extended by a factor from $2.29 \mathrm{x}$ to $4.82 \mathrm{x}$ and the overall cycle efficiency is improved from $11.9 \%$ to $21.9 \%$, compared to the baseline system. For the sinusoid source and load profile, the cycle life enhancement factor varies from $1.76 \mathrm{x}$ to $3.66 \mathrm{x}$, and cycle efficiency improvement varies from $5.0 \%$ to $12.9 \%$.

\section{CONCLUSION}

This paper introduces the state of health $(\mathrm{SoH})$-aware charge management problem for hybrid electrical energy storage (HEES) system for the first time. We propose an efficient algorithm aiming at extending the cycle life of battery arrays as well as improving the overall cycle efficiency. We first apply a crossover filter to the power profiles of source /load and let the supercapacitor bank deal with the high frequency component of the power supple or demand. Then we charge or discharge supercapacitor bank accordingly to maintain the desired operation conditions for the battery bank. The proposed algorithm effectively reduces the average state of charge (SoC) swing and average SoC level of the battery array, which is proven helpful in extending battery bank cycle life. To improve the cycle efficiency in HEES system, we solve the optimal target CTI voltage and operation currents for EES banks using ternary search. Experimental results show significant performance improvement up to $21.9 \%$ and $4.82 \mathrm{x}$ in terms of overall cycle efficiency and battery array cycle life, respectively.

\section{REFERENCES}

[1] M. Pedram, N. Chang, Y. Kim, and Y. Wang, "Hybrid electrical energy storage systems," in ISLPED, 2010.

[2] F. Koushanfar, "Hierarchical hybrid power supply networks," in $D A C$, 2010.

[3] Y. Wang, Y. Kim, Q. Xie, N. Chang, and M. Pedram, "Charge migration efficiency optimization in hybrid electrical energy storage (HEES) systems," in ISLPED, 2011.

[4] Q. Xie, Y. Wang, Y. Kim, N. Chang and M. Pedram, "Charge Allocation for Hybrid Electrical Energy Storage Systems," CODES+ISSS, 2011.

[5] G. Sikha, R. Ramadass, B. Haran, R. Whiteand, and B. Popov," Comparison of the capacity fade of Sony US18650 cells charged with different protocols", Journal of Power Sources, 122 (2003) 67.

[6] J. Li, E. Murphy, J. Winnick, and P. Kohl, "The effects of pulse charging on cycling characteristics of commercial lithium-ion batteries", Journal of Power Sources, 102 (2001) 302.

[7] S. Zhang, "The effect of the charging protocol on the cycle life of a Liion battery", Journal of Power Sources, 161 (2006) 1385.

[8] Y.Liu, C. Hsieh, Y. Luo, "Search for an Optimal Five-Step Charging Pattern for Li-Ion Batteries Using Consecutive Orthogonal Arrays," IEEE Trans. on Energy Conversion, vol.26, no.2, pp.654-661.

[9] A. Millner, "Modeling Lithium Ion Battery Degradation in Electric Vehicles," in CITRES, 2010.

[10] J. Belt, C. Ho, C. Motloch, T. Miller and T. Duong, "A capacity and power fade study of Li-ion cells during life cycle testing", Journal of Power Sources, 123 (2003) 241-246.

[11] M. Dubarry, V. Svoboda, R. Hwu and B.Liaw, "Capacity and power fading mechanism identification from a commercial cell evaluation", Journal of Power Sources, 2007.

[12] S. Peterson, J. Apt and J. Whitacre, "Lithium-ion battery cell degradation resulting from realistic vehicle and vehicle-to-grid utilization", Journal of Power Sources, 2010.

[13] P. Ramadass, B. Haran. R. White and B. Popov, "Mathematical modeling of the capacity fade of Li-ion cells", JPS, 2003.

[14] Q. Zhang and R. White, "Capacity fade analysis of a lithium ion cell", Journal of Power Sources, 179 (2008) 793-798.

[15] P. Rong and M. Pedram, "An analytical model for predicting the remaining battery capacity of Lithium-ion batteries", IEEE TVLSI, 2006.

[16] M. Chen and G. Rincon-Mora, "Accurate electrical battery model capable of predicting runtime and I-V performance," TEC, 2006.

[17] D. Shin, and et al, "Battery-supercapacitor hybrid system for high-rate pulsed load applications," in DATE, 2011.

[18] D. Linden and T. B. Reddy, Handbook of Batteries. McGrew-Hill Professional, 2001

[19] Y. Choi, N. Chang and T. Kim, "DC-DC converter-aware power management for low-power embedded systems," TCAD, 2007. 
